\title{
Desenvolvimento de software para interpretação de dados gasométricos aplicável em unidades de terapia intensiva
}

Software development for gasometric data interpretation applicable in intensive care units Tiana Oliveira Vidal Fernandes', Rodrigo Santos de Queiroz ${ }^{2}$, Ramon Santana de Jesus ${ }^{3}$, Lucas Silva Vasconcelos ${ }^{4}$

RESUMO I O objetivo deste estudo foi desenvolver um software para interpretação de dados gasométricos aplicável em UTIs. Trata-se de estudo de caráter experimental, sendo selecionada uma base teórica em Java com a IDE NetBeans 6.8 por meio de parceria com profissionais capacitados em Sistemas de Informação. O desenvolvimento do programa foi baseado na criação de um algoritmo, uma sequência de instruções bem definidas e não ambíguas a serem executadas mecanicamente com a finalidade de fornecer um diagnóstico desejado. Foi criado um software aplicável em UTIs denominado InterGas, que é um programa de fácil instalação, possui interface de fácil compreensão e utilização, além de processar os dados rapidamente e de forma precisa, oferecendo como resultado final o diagnóstico para o distúrbio do equilíbrio ácido-básico. O desconhecimento de outra ferramenta que reúna todos os componentes do InterGas o torna um software pioneiro que facilita a tomada de decisão à medida que caracteriza a ocorrência de distúrbios mistos utilizando fórmulas de compensação. Com isso, futuros estudos deverão ser feitos com o objetivo de avaliar aspectos relacionados à implementação e eficácia do software desenvolvido.

Descritores I software; gasometria; Unidades de Terapia Intensiva; tecnologia biomédica; diagnóstico.

\begin{abstract}
I The objective of this study was to develop a software for data interpretation of gasometric applicable in Intensive Care Units that can provide a diagnosis for disorders of acid-base balance. This is an experimental study being selected on a theoretical basis with the Java IDE NetBeans 6.8 through a partnership with professionals trained in Information Systems. The program development was based on creating an algorithm, a sequence of well-defined and unambiguous to be performed mechanically in order to provide a desired diagnosis. We created a software applicable in intensive care units called InterGas, which is a program easy to install, has an interface easy to understand and use, and to process the data quickly and accurately providing as the final diagnosis for the disorder the acid-base balance. The lack of another tool that brings together all the components of InterGas makes it a pioneering software that facilitates decision-making, as it characterizes the occurrence of mixed disturbances using compensation formulas.
\end{abstract}

Keywords | software; blood gas analysis; Intensive Care Units; biomedical technology; diagnosis.

Estudo desenvolvido na Universidade Estadual do Sudoeste da Bahia (UESB) - Jequié (BA), Brasil.

'Fisioterapeuta pela UESB - Jequié (BA), Brasil.

2Professor do curso de Fisioterapia da UESB - Jequié (BA), Brasil.

${ }^{3}$ Acadêmico do curso de Fisioterapia da UESB - Jequié (BA), Brasil.

${ }^{4}$ Acadêmico do curso de Sistemas de Informação da Universidade Salvador (UNIFACS) - Salvador (BA), Brasil. 


\section{INTRODUÇÃO}

A Gasometria do Sangue Arterial (GSA), ou análise de gases no sangue arterial, é um exame realizado rotineiramente em pacientes submetidos à anestesia ou internados em Unidade de Terapia Intensiva (UTI), sendo suas principais indicações a avaliação do distúrbio do equilíbrio ácido-base, da oxigenação pulmonar do sangue arterial e da ventilação alveolar ${ }^{1}$. A GSA tem por objetivo revelar valores de $\mathrm{pH}$ sanguíneo, da pressão parcial de gás carbônico $\left(\mathrm{PaCO}_{2}\right)$ e oxigênio $\left(\mathrm{PaO}_{2}\right)$, íon bicarbonato $\left(\mathrm{HCO}_{3}\right)$ e saturação da oxi-hemoglobina, dentre outros ${ }^{2,3}$. O resultado da gasometria é utilizado para avaliação de doenças respiratórias e de outras condições que afetem os pulmões, bem como fornece informações a respeito de doenças metabólicas mediante a interpretação do funcionamento dos sistemas tampão orgânicos ${ }^{1,2}$.

Diante de um distúrbio ácido-base é sempre importante buscar o diagnóstico etiológico, a fim de que a abordagem terapêutica seja dirigida à causa básica, sendo fundamental a caracterização do tipo de distúrbio apresentado pelo paciente. Existem algumas regras que podem ser utilizadas para avaliar as respostas compensatórias aos distúrbios ácido-base que envolvem a utilização de fórmulas e cálculos matemáticos que demandam tempo para a sua realização e, portanto, nem sempre são realizados nas Unidades de Terapia Intensiva. Dessa forma, a interpretação dos dados gasométricos parece ser incompleta, ou até mesmo equivocada, quando não se realiza a análise da possibilidade de presença de distúrbio misto e limita-se ao tratamento de um distúrbio simples ${ }^{1,4}$.

A aplicação da informática na área da saúde é fato concreto e encontra-se em desenvolvimento acelerado, contribuindo para mudanças nas estruturas organizacionais dos hospitais ${ }^{5}$. Segundo Novaes ${ }^{6}$, a área de avaliação de programas, serviços e tecnologias em geral e na saúde, em particular, passa por um processo de expansão e diversificação conceitual e metodológica, bem como por uma crescente demanda para se constituir em instrumento de apoio às decisões necessárias à dinâmica dos sistemas e serviços de saúde. A revolução da informação, com o advento dos computadores presentes na nossa vida diária, vem provocando mudanças nos hábitos e valores de vida. Atualmente, muitas empresas investem em Tecnologia da Informação visando à resolução de problemas, em praticamente todas as áreas onde um ser humano possa atuar, por meio de um software que é definido como conjunto de instruções (programa de computador) que, quando executadas, produzem função ou resultado satisfatório ${ }^{7}$. Assim, um projeto de desenvolvimento de software pode começar quando alguém sugere que uma nova função automática é necessária ${ }^{8}$.

Nesse contexto, percebe-se a necessidade de desenvolver um programa eficaz, eficiente, prático e acessível à UTI, que gere um diagnóstico correto e completo a partir dos dados obtidos na GSA de forma instantânea, otimizando o atendimento aos pacientes críticos, visto que a interpretação fidedigna dos resultados das gasometrias fornece subsídios para a interpretação da clínica e tomada de decisão, como possíveis alterações de parâmetros de ventilação mecânica e/ou suplementação de oxigênio, dentre outras terapêuticas, dessa forma auxiliando os profissionais responsáveis, sobretudo o fisioterapeuta, na escolha da correta terapêutica a ser utilizada, visto que esta deve ser sempre baseada na real situação clínica do paciente, sendo imprescindível para a recuperação do paciente em UTI.

Diante disso, o presente estudo teve como objetivo desenvolver um software para Interpretação Gasométrica (InterGas) capaz de fornecer um diagnóstico para os distúrbios do equilíbrio ácido-básico, quando presentes, a partir dos cálculos propostos pelas três etapas iniciais do "Primeiro Método de Análise" descrito por Rocco ${ }^{1}$.

\section{MÉTODOS}

Foi utilizado o "Primeiro Método de Análise" proposto por Rocco que consiste em avaliar a existência de distúrbio ácido-base em três etapas, são elas: verificar a validade da gasometria arterial utilizando a equação de Henderson-Hasselbalch $(\mathrm{H}-\mathrm{H})$, verificar qual o distúrbio primário e verificar se existe distúrbio secundário ${ }^{1}$. Trata-se de um estudo de caráter experimental o qual foi utilizado como base teórica para nortear o desenvolvimento do software, tendo em vista a ausência de um consenso nacional ou um documento oficial sobre métodos de análise hemogasométrica.

Após a escolha do método de análise de gasometria, o InterGas foi desenvolvido usando o modelo evolucionário da engenharia de software, que propõe a participação ativa do usuário durante o processo de desenvolvimento, assegurando um produto adequado às necessidades do cliente. Nesse modelo, as etapas de especificação, desenvolvimento e validação ocorrem de forma concorrente 9 . Além disso, o desenvolvimento do InterGas buscou contemplar as recomendações do programa para Melhoria 
de Processo do Software Brasileiro (MPS.BR), coordenado pela Associação para Promoção da Excelência do Software Brasileiro (SOFTEX), contando com o apoio do Ministério da Ciência e Tecnologia (MCT), da Financiadora de Estudos e Projetos (FINEP) e do Banco Interamericano de Desenvolvimento (BID) $)^{10}$

O programa foi desenvolvido na linguagem de programação Java com a IDE NetBeans 6.8 que é uma ferramenta gratuita, sem restrição de uso, de código aberto, escrita inteiramente em Java, desenhada para escrever, compilar, depurar e executar programas com ambiente de desenvolvimento integrado que permite a criação de aplicativos de forma independente e que podem ser entendidos facilmente por outros desenvolvedores de software ${ }^{11}$.

As fases da engenharia de software foram identificadas segundo Pressman ${ }^{9}$, servindo como roteiro para o desenvolvimento do InterGas. A primeira fase de análise de requisitos levou à avaliação da viabilidade do software e estabelecimento dos requisitos para o seu desenvolvimento: usabilidade, funcionalidade, fácil instalação, fácil manuseio, processamento rápido e preciso dos dados com resultado final de diagnóstico para o distúrbio ácido-básico de cada paciente de acordo com os dados informados pelo usuário, e fácil acesso em UTI por meio de computadores.

Os dados de entrada (input) que deverão ser fornecidos ao software na sua utilização serão: $\mathrm{pH}, \mathrm{PaCO}_{2}$, $\mathrm{HCO}_{3}$ e caracterização do quadro do paciente em agudo ou crônico. Já os dados de saída (output), que são, nesse caso, as informações diagnósticas geradas pelo software, contemplarão todos os tipos de distúrbios do equilíbrio ácido-base, diferenciando-os em simples ou mistos, bem como serão identificadas as gasometrias não validadas de acordo com a equação de $\mathrm{H}-\mathrm{H}$ e os casos de resultados gasométricos normais sem a presença de distúrbio ácido-base.

Nas fases de Definição e Projeto, foi elaborado um roteiro para ser fornecido aos profissionais de sistemas de informação que continha uma sequência de cinco passos a serem seguidos pelo InterGas (Quadro 1) com os cálculos necessários para a identificação do diagnóstico correto para o distúrbio apresentado a fim de guiar a fase de Implementação marcada pela criação do software, por parte dos programadores (Quadro 1).

O desenvolvimento do programa foi baseado na criação de um algoritmo, constituindo-se numa sequência de instruções bem definidas e não ambíguas a serem executadas mecanicamente com a finalidade de fornecer um diagnóstico desejado, ou seja, foi criado um roteiro com estruturas condicionais apresentando possibilidades que levam ao diagnóstico correto, seguindo um passo a passo e realizando comparações lógicas quando necessário ${ }^{12}$.

Após a integração (construção) do software, um conjunto de testes foi realizado, utilizando uma suíte de testes unitários (JUnit), e os critérios de validação que foram estabelecidos na fase de análise de requisitos foram testados, oferecendo a garantia final de que o programa atende a todas as exigências funcionais, comportamentais e de desempenho. Nessa fase (Fase de Testes e Validação), foram testadas todas as possibilidades diagnósticas existentes que deveriam ser identificadas pelo InterGas com exemplos de dados gasométricos que representassem cada um dos diagnósticos a seguir: Gasometria Inválida, Gasometria Normal, Acidose Metabólica (AcM), Acidose respiratória aguda (AcRA), Acidose respiratória crônica (AcRC), Acidose mista, Alcalose metabólica (AM), Alcalose respiratória aguda (ARA), Alcalose respiratória crônica (ARC), Alcalose mista, AcRA + AM, AcRC + AM, AcM + alcalose respiratória, ARA + AcM, ARC + AcM, AM + acidose respiratória.

\section{RESULTADOS E DISCUSSÃO}

Foi desenvolvido um software denominado InterGas capaz de interpretar dados gasométricos e que pode ser

Quadro 1. Roteiro fornecido aos profissionais de Sistemas de Informação para configuração sequencial do software

$\begin{array}{ll}1^{\circ} \text { passo: } & \text { validação da gasometria de acordo com a equação de } \mathrm{H}-\mathrm{H} . \\ 2^{\circ} \text { passo: } & \text { identificação do distúrbio ácido-base primário baseado nos valores normais de } \mathrm{PaCO}_{2}, \mathrm{HCO}_{3} \text { e } \mathrm{pH} \text { sanguíneo, considerando alcalemia e acidemia. } \\ 3^{\circ} \text { passo: } & \begin{array}{l}\text { cálculo da variação de } \mathrm{PaCO}_{2} \text { e } \mathrm{HCO}_{3} \text { apresentadas pelos dados gasométricos fornecidos quando comparados aos valores normais, que } \\ \text { étil à avaliação da presença de distúrbio secundário. }\end{array} \\ 4^{\circ} \text { passo: } & \begin{array}{l}\text { avaliação da presença de distúrbio secundário por meio de cálculos baseados nas fórmulas de compensação descritas por Rocco' } \\ \text { distintas para cada distúrbio primário identificado no segundo passo. }\end{array} \\ 5^{\circ} \text { passo: } & \begin{array}{l}\text { comparação entre a variação de } \mathrm{PaCO}_{2} \text { e } \mathrm{HCO}_{3} \text { fornecidos e os valores de variação esperados para cada gasometria permitindo a } \\ \text { identificação do distúrbio secundário quando presente. }\end{array}\end{array}$

H-H: Henderson-Hasselbalch; $\mathrm{PaCO}_{2}$ : pressão arterial de dióxido de carbono; $\mathrm{HCO}_{3}$ : concentração sérica de bicarbonato; pH: potencial de hidrogênio. 
utilizado em diversos ambientes, inclusive UTIs, onde pode ser de grande utilidade por otimizar o tempo e permitir um diagnóstico correto, facilitando o planejamento e a tomada de decisão. O InterGas é de fácil instalação, possui uma interface de simples compreensão, facilitando a sua utilização, além de processar os dados rapidamente e de forma precisa, oferecendo como resultado final o diagnóstico para o distúrbio ácido-base de cada paciente de acordo com os dados informados pelo usuário. Possui uma tela principal na qual podem ser identificados quatro campos a serem preenchidos que correspondem ao $\mathrm{pH}, \mathrm{PaCO}_{2}, \mathrm{HCO}_{3}$ e caracterização do quadro clínico apresentado pelo paciente em agudo ou crônico. Logo abaixo o usuário tem a opção de clicar nos botões "Diagnóstico", para obter o diagnóstico do distúrbio ácido-base, ou "Limpar", para permitir uma nova consulta com a inserção de novos dados (Figura 1).

Após o clique no botão "Diagnóstico", o software fornece imediatamente o resultado solicitado no campo que fica abaixo desse botão, apresentando apenas o diagnóstico referente aos dados inseridos que são processados por meio da realização dos cálculos, para os quais são utilizadas até três casas decimais. A Figura 2 ilustra o resultado da interpretação dos seguintes dados gasométricos: $\mathrm{pH}=7.29, \mathrm{PaCO}_{2}=61, \mathrm{HCO}_{3}=29$ e quadro clínico agudo (Figura 2).

A seguir, no Quadro 2, temos os mesmos dados gasométricos mostrados na figura anterior (Figura 2) sendo interpretados manualmente e com auxílio da calculadora científica (Quadro 2).

A comparação entre o Quadro 2 e a Figura 2 evidencia a praticidade do software visto a extensão dos cálculos, demanda de tempo e domínio do assunto exigido por parte do profissional de saúde que deve organizar um raciocínio matemático para então iniciar a realização dos cálculos, necessitando ainda dispor de calculadora científica acessível no momento. Assim, o InterGas parece otimizar o tempo, visto que, tendo o software ao seu alcance, o profissional apenas deve preencher quatro campos solicitados e clicar no botão "Diagnóstico", garantindo uma visão mais completa do real distúrbio do equilíbrio ácido-básico apresentado pelo paciente de forma rápida.

Além disso, sabe-se que a dificuldade de realização dos cálculos muitas vezes limita a interpretação da gasometria à identificação do distúrbio primário, o que leva a uma consequente limitação nas condutas e no tratamento. Considerando-se que os distúrbios do equilíbrio ácido-base são comuns nos pacientes críticos, estando em geral associados à maior morbimortalidade,

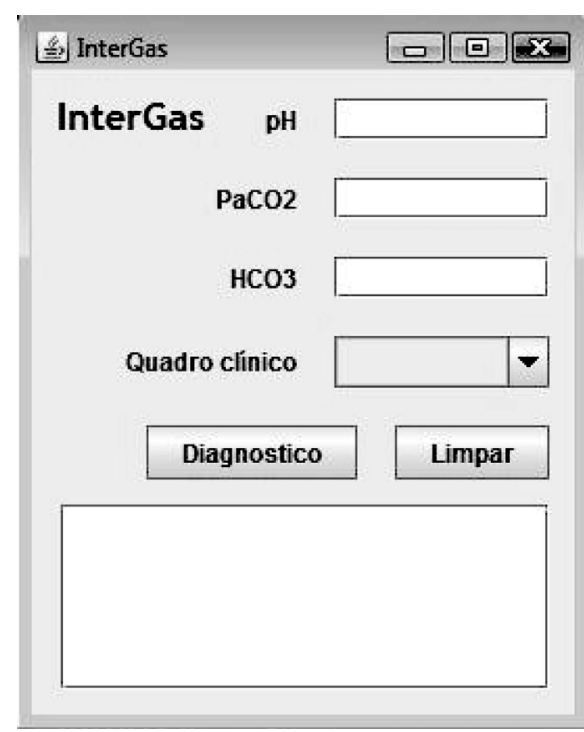

pH: potencial de hidrogênio; $\mathrm{PaCO}$ : pressão arterial de dióxido de carbono; $\mathrm{HCO}_{3}$ : concentração sérica de bicarbonato.

Figura 1. Tela principal do InterGas

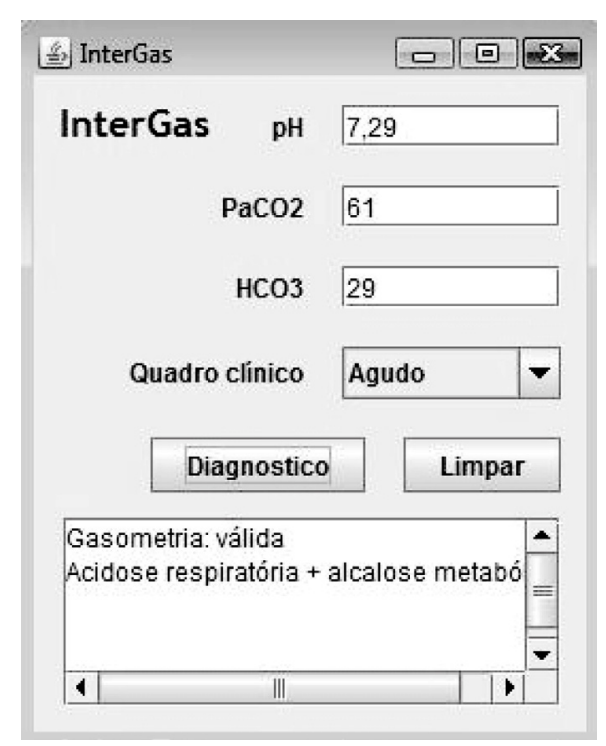

pH: potencial de hidrogênio; $\mathrm{PaCO}$ : pressão arterial de dióxido de carbono; $\mathrm{HCO}_{3}$ : concentração sérica de bicarbonato.

Figura 2. Apresentação diagnóstica do software InterGas a partir dos dados gasométricos inseridos

métodos que permitam identificar atitudes que possam evitá-los e/ou corrigi-los são, portanto, extremamente importantes ${ }^{13}$.

O software desenvolvido constitui-se numa ferramenta útil para a interpretação de dados gasométricos, caracterizando a ocorrência de distúrbios do equilíbrio ácido-base, simples ou mistos. Grogono ${ }^{14}$ criou o "Acid-Base Diagram" que é uma ferramenta interativa, disponível na Internet, capaz de informar diagnóstico de distúrbios do equilíbrio ácido-básico a partir de dados gasométricos; entretanto, segundo o próprio autor, o programa não possui versão transferível e está 
Quadro 2. Base do raciocínio matemático implementado pelo InterGas para gerar diagnóstico hemogasométrico

\section{0 passo: validação pela equação de $\mathrm{H}-\mathrm{H}$ : \\ $\mathrm{pH}=6.10+\log \left(\left[\mathrm{HCO}_{3}\right] /\left[\mathrm{PaCO}_{2} \times 0.03060\right]\right)$ \\ $\mathrm{pH}=6.10+\log ([29] /[61 \times 0.03060])$ \\ $\mathrm{pH}=6.10+\log ([29] /[1.8666])$ \\ $\mathrm{pH}=6.10+\log (15.5363)$ \\ $\mathrm{pH}=6.10+1.19$ \\ $\mathrm{pH}=7.29$}

$2^{\circ}$ passo: verificação do distúrbio ácido-base primário:

Acidose respiratória identificada a partir da comparação com os

valores normais de $\mathrm{pH}, \mathrm{PaCO}_{2}{\mathrm{e} \mathrm{HCO}_{3}}$

$3^{\circ}$ passo: cálculo da variação de $\mathrm{PaCO}_{2}$ e $\mathrm{HCO}_{3}$

$\Delta \mathrm{PaCO}_{2}=\left[40-\mathrm{PaCO}_{2}\right.$ fornecido $]$

$\triangle \mathrm{PaCO}_{2}=[4 \mathrm{O}-61]$

$\triangle \mathrm{PaCO}_{2}=21$

$\Delta \mathrm{HCO}_{3}=\left[24-\mathrm{HCO}_{3}\right.$ fornecido $]$

$\Delta \mathrm{HCO}_{3}=[24-29]$

$\Delta \mathrm{HCO}_{3}=5$

$4^{\circ}$ passo: aplicação da fórmula de compensação para o distúrbio primário identificado:

Fórmula de compensação para acidose respiratória aguda:

$\Delta \mathrm{HCO}_{3}$ esperada $=0.1-0.2 \times \Delta \mathrm{PaCO}_{2}$

$\Delta \mathrm{HCO}_{3}$ esperada $=0.1-0.2 \times 21$

$\Delta \mathrm{HCO}_{3}$ esperada $=2.1-4.2$

$5^{\circ}$ passo: comparação entre a variação de bicarbonato encontrada $\left(\triangle \mathrm{HCO}_{3}\right)$ e a esperada $\left(\triangle \mathrm{HCO}_{3}\right.$ esperada):

A $\Delta \mathrm{HCO}_{3}$ foi maior do que a $\Delta \mathrm{HCO}_{3}$ esperada, o que indica a presença de alcalose metabólica associada.

Logo, o diagnóstico é: Acidose Respiratória + Alcalose Metabólica.

$\mathrm{H}-\mathrm{H}$ : Henderson-Hasselbalch; $\mathrm{PaCO}_{2}$ : pressão arterial de dióxido de carbono; $\triangle \mathrm{PaCO}$ : variação pressão arterial de dióxido de carbono; $\mathrm{HCO}_{\text {: }}$ concentração sérica de bicarbonato; $\triangle \mathrm{HCO}$ : variação da concentração sérica de bicarbonato; $\mathrm{pH}$ : potencial de hidrogênio; log: função logarítmica.

disponível apenas no site. Logo, a necessidade de conexão com a Internet reduz a sua usabilidade dentro da UTI, bem como a sua interface, na qual o usuário precisa encontrar os dados que deseja interpretar nas linhas de um gráfico por meio de cliques em áreas pequenas que variam os valores numéricos com pequenas variações na área clicada, criando uma situação de tentativa e erro até encontrar os dados precisos, enquanto no InterGas esses dados aferidos da GSA do paciente são facilmente inseridos pelo usuário.

O desconhecimento de outra ferramenta que reúna todos os componentes do InterGas o torna um software pioneiro que merece ser aperfeiçoado e melhor avaliado para que possa ser utilizado no ambiente operacional para o qual foi desenvolvido (UTI). Dessa forma, vale ressaltar que a validação do InterGas está em conformidade com processos de normas de desenvolvimento de software em sua utilização e avaliação fora do ambiente operacional. Entretanto, ainda deve passar por uma fase de utilização e aplicação dentro da UTI para estar de acordo com o processo de validação da MR-MPS ${ }^{10}$.
Constitui-se em recomendação para estudos futuros a avaliação de margens de erro, de confiabilidade e usabilidade do InterGas mediante a utilização e avaliação do programa por profissionais intensivistas diante de situações reais na UTI. Outra recomendação é a implementação de métodos específicos de análise para cada tipo de distúrbio, incluindo os valores de Base Excess (BE) e o cálculo de GAPS (anion-gap, delta anion-gap, anion-gap urinário e gap-osmolar) proposto na quarta etapa do primeiro método de análise de Rocco ${ }^{1}$. Dentre outros métodos de análise, os que incluem o cálculo do "Standard Base Excess" e o "método de Stewart" descritos por Rocco ${ }^{1}$ como o segundo e o terceiro métodos, respectivamente, sendo este último citado como padrão-ouro em algumas temáticas ${ }^{14-20}$, mas impossibilitado de utilização por este trabalho por conta da rotina da UTI em pesquisa em relação aos exames laboratoriais, que muitas vezes não são requisitados por completo, impossibilitando a utilização do mesmo.

Demais sugestões para estudos futuros levam em consideração o desenvolvimento de uma versão para o InterGas que possa ser utilizada em dispositivos móveis, como celulares e palmtops, e a possibilidade de gerar informações mais complexas, como sugerir hipóteses de tratamento, alteração de parâmetros de ventilação mecânica e outras terapêuticas, como infusão lenta de bicarbonato, por exemplo.

Foram identificados erros e necessidade de ajustes e reconstrução de alguns passos para ser realizada uma nova fase de Testes e Validação, que é essencialmente uma verificação da função do software com o objetivo principal de confirmar que todos os requisitos foram cumpridos $^{8}$. Após essa fase, o InterGas forneceu corretamente os diagnósticos para todos os tipos de distúrbio esperados, bem como para os resultados normais de gasometrias.

\section{CONCLUSÃO}

Foi desenvolvido o software InterGas, de fácil instalação que possui interface de simples compreensão e utilização, além de processar os dados rapidamente e de forma precisa, oferecendo como resultado final o diagnóstico para o distúrbio do equilíbrio ácido-básico. O desconhecimento de outra ferramenta que reúna todos os componentes do InterGas o torna um software pioneiro que facilita a tomada de decisão à medida que caracteriza a ocorrência de distúrbios mistos utilizando fórmulas 
de compensação. Necessita ainda de mais estudos que possam aperfeiçoá-lo dentro das UTIs, possibilitando a análise hemogasométrica mais fidedigna e amparando a tomada de decisão pelos profissionais de saúde com uma maior praticidade e confiabilidade.

\section{REFERÊNCIAS}

1. Rocco JR. Diagnóstico dos distúrbios do metabolismo ácido-base. Rev Bras de Ter Intensiva. 2003:15(4):184-92.

2. Atherton JC. Acid-base balance: maintenance of plasma $\mathrm{pH}$. Anaesthesia and Intensive Care Medicine. 2006;7(11):427-31.

3. Zimmerman JL, Dellinger RP. Blood gas monitoring. Crit Care Clin. 1996:12(4):865-74

4. Scanlan CL, Wilkins RL, Stoller, JK. Fundamentos da Terapia Respiratória de Egan. 7a ed. São Paulo: Manole; 2000.

5. Barbosa MBG, Alves CAD, Filho HQ. Avaliação da acidose metabólica em pacientes graves: método de Stewart-Fencl-Figge versus a abordagem tradicional de Henderson-Hasselbalch. Rev Bras de Ter Intensiva. 2006:18(4):380-4.

6. Novaes HMD. Avaliação de programas, serviços e tecnologias em saúde. Rev Saúde Pública. 2000;34(5):547-59.

7. Dalri MCB, Carvalho EC. Planejamento da assistência de enfermagem a pacientes portadores de queimadura utilizando um software: aplicação em quatro pacientes. Rev Latino-am Enfermagem. 2002:10(6):787-93.

8. Prado AA, Silva MB, Ribeiro RB, Rosa JL. Engenharia de software em aplicações de tecnologia da informação visando maior qualidade nos sistemas de informações gerenciais. Rev Janus. 2006;3(3).
9. US. Department of Health and Human Services; Food and Drug Administration; Center for Devices and Radiological Health; Center for Biologics Evaluation and Research. General Principles of Software Validation; Final Guidance for Industry and FDA Staff, January 11, 2002.

10. Pressman, RS. Engenharia de Software. 6a. ed. São Paulo: McGrawHill; 2006

11. SOFTEX. MPS.BR - Melhoria de Processo do Software Brasileiro. Guia Geral V1.1-Maio/2006.

12. Myatt A. Netbeans IDE 6 Pro Edition Rich Client Platform. Berkeley: Apress; 2008.

13. Cormen TH, Leiserson CE, Rivest RL. Algoritmos - Teoria e Prática. 2a ed. Rio de Janeiro: Campus; 2002.

14. Grogono AW. Acid-Base Tutorial. New Orleans: Tulane University Department of Anesthesiology; [Updated 2010 Mar 12; cited 2012 Apr 1]. Available from: http://www.acid-base.com/diagram.php.

15. Boniatti MM, Cardoso PRC, Moraes RB. Distúrbios ácido-básicos em pacientes críticos - método de Stewart. Scientia Medica, Porto Alegre: PUCRS; 2006:16(2)

16. Sirker AA, Rhodes A, Grounds RM, Bennett ED. Acid-base physiology: the 'traditional' and the 'modern' approaches. Anaesthesia. 2002;57:348-56

17. Rastegar A. Clinical utility of Stewarts method in diagnosis and management of acid-base disorders. Clin J Am Soc Nephrol. 2009:4(7):1267-74.

18. Dubin A, Menises MM, Masevicius FD, Moseinco MC, Kutscherauer DO, Ventrice $E$, et al. Comparison of three different methods of evaluation of metabolic acid-base disorders. Crit Care Med. 2007;35(5):1264-70.

19. Noritomi DT, Sanga RR, Amaral ACK-B, Park M. Metabolic acid-base status in critically ill patients: is standard base excess correlated with serum lactate level? Rev Bras de Ter Intensiva. 2006;18(1):22-6.

20. Évora PRB, Garcia LV. Equilíbrio ácido-base. Medicina (Ribeirão Preto). 2008:41(3):301-11. 